\section{BALL ON MECHANICS*}

THE object of this book is to "prove the elementary laws of mechanics by means of experiments"-a method the exact opposite of that generally adopted. According to the usual method, a few very general principles are assumed as derived from experimental data, a group of intermediate principles is then obtained deductively, by the aid of which the action of forces in particular cases can be analysed. The particular cases may be such as have an interest from their bearing on practical questions, but they are only examples of a general method applicable to innumerable other cases. There are therefore two distinct objects for which mechanical experiments may be made-viz, either to verify the fundamental principles, or to verify the deductions drawn in particular cases. Experiments of the former kind are absolutely essential to the existence of the science. Unless, for instance, the conditions of the action of the force of friction are determined by experiment, no deductions as to cases into which that force enters have any but a theoretical value. The same is true in all similar cases; such questions as, whether quantity of matter is proportional to weight, whether gravity at a given station is sensibly a constant force, whether the elasticity of solid bodies follows Hooke's law, and if so within what limits, can be answered by experiment only. Such questions, on the other hand, as the tension of a tie-rod under given circumstances, the relation between the weights which keep a given lever at rest,

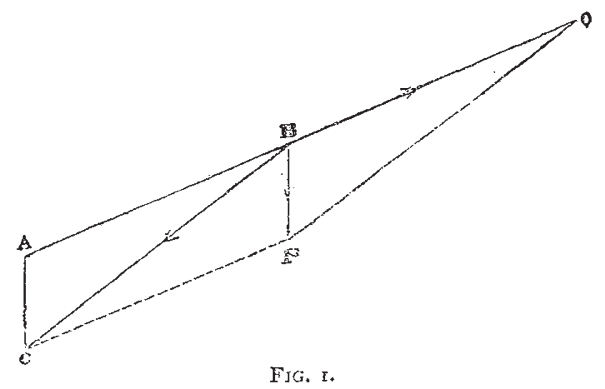

the relation between the power and the weight in a block and tackle, the form of the surface of a revolving liquid, admit of exact answers by deduction from the proper data, and, of course, the answers may be tested by experiment. Such experiments clearly have a different object from those of the former class. They have, indeed, this in common, that experiments of the latter kind also serve to verify fundamental principles, but they o o so indirectly. It is, however, from the teacher's point of view that their value will be found greatest. In teaching the elementary parts of mechanics perhaps the greatest difficulty experienced is to make the learner feel that the diagrams drawn on the black board represent facts, that, for instance, the conclusion deduced from a triangle is really applicable to a crane. Put the experiment side by side with the deduction, and it will be seen that the experiment cannot fail to bring home to the mind of the learner that his reasoning relates to things and not merely to abstractions.

Let $C B$ (Fig. I) represent the jib or strut, and $A B$ the tierod of a crane, the line $A C$ being vertical. Let a weight $P$ hang from $\mathrm{A}$, and let it be required to determine the forces transmitted through the tie and the jib. $\mathrm{P}$ can be resolved into two forces acting along $\mathrm{BC}$ and $\mathrm{AB}$ produced, and an inspection of the figure will show that these forces bear to $\mathrm{P}$ the same ratio that the lines $\mathrm{BC}$ and $\mathrm{AB}$ bear to $A C$, and that the force along $B C$ is a thrust, and that along $\mathrm{AB}$ a tension. This analysis is perfectly general.

* Experimental Mechunics : a Contrse of Lectures delizicred at the Royat College of Science for Treland. By Robert Stawell Ball, M.A. With Illus trations. (London and New York: Macmillan and Co., 187r.)
We will now give Mr. Ball's experiment in illustration of the same question :- "A piece of pine $\mathrm{BC}, 3^{\prime} 6^{\prime \prime}$ long and $I^{\prime \prime} \times I^{\prime \prime}$ in section ( $\mathrm{Fig}$. 2 ) is capable of turning round its support at the bottom $B$ by means of a joint or hinge; it is held up by a tie AC $3^{\prime}$ long, which is attached to the support exactly above the joint. $A B$ is $x^{\prime}$ long. From the point $\mathrm{C}$ a wire descends, having a hook at the end, on which a weight can be hung. The tie is attached to the spring balance, the index of which shows the strain. The spring balance is supported by a wire strainer, by turning the nut of which the length of the wire can be shortened or lengthened as occasion requires. This is necessary, because when different weights are suspended from the hook the spring is stretched more or less, and the screw is then employed to keep the entire length of the tie at $3^{\prime}$. The remainder of the tie consists of copper wire" (p. 29). Mr. Ball then goes on to notice that when a weight of 2olbs. is placed on the hook, the strain, as determined by the spring balance, is 6olbs., thus verifying the analysis of the case given above.

As an example of an experiment of the former class we will take the following,-it is the form in which Mr. Ball

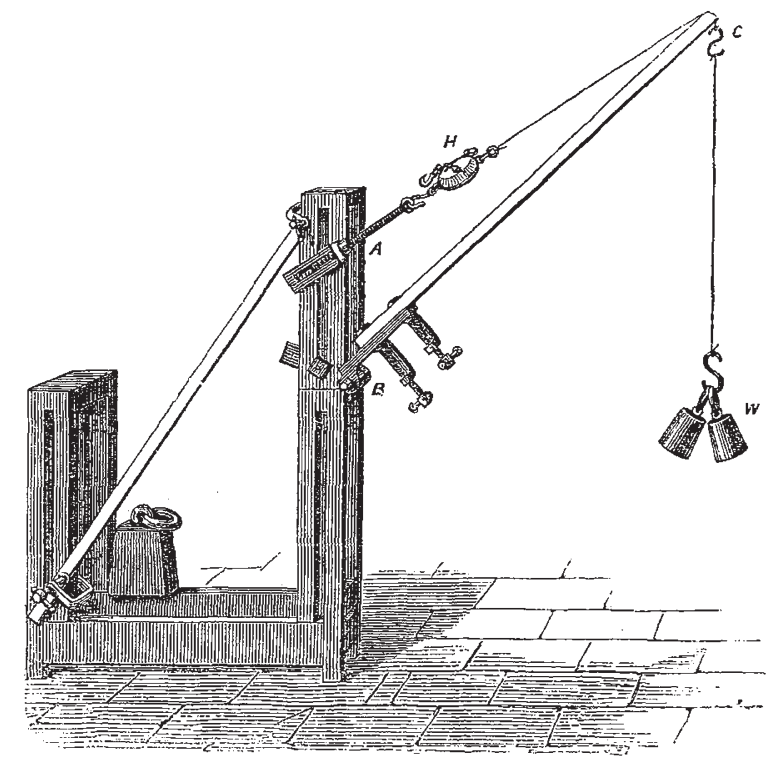

FIG.2.

gives Galileo's experiment of dropping bodies from the top of the Tower of Pisa. The figure (Fig. 3 ) is so perfect that it scarcely requires explanation. Solong as the current is in action, the horse-shoe $G$ is magnetic, and a ball of iron $F$ remains suspended from it. When the current is broken $G$ is no longer magnetic and $F$ falls. In this manner, by including the wire round both horse-shoes in the circuit, a ball of iron and one of wood, into which a flat-headed nail has been driven, can be kept suspended, and then by breaking the circuit they can be let fall at exactly the same instant, they are seen to reach the cushion at the same instant, and are thus shown to fall through equal spaces in the same time. Mr. Ball describes and discusses the experiment at some length, and shows how it proves that at a given station the attraction of gravitation on different bodies is proportional to their masses.

The above examples will give a better notion both of the contents and illustrations of the book than any long description. We may say, however, that the book contains a clear and correct exposition of the first principle; of mechanics, and illustrates, by well-chosen experiments, all the points in the subject that can be fairly called elementary. The figures reproduce all the circumstances of the experiments with so much exactness that with 
moderate care the reader will understand the points illustrated nearly as well as if he saw the experiments themselves. In great part Mr. Ball has devised these experiments himself, and thus in the well-worked field of elementary mechanics he has introduced much that is original in treatment, and in some parts-particularly in his lecture on friction-there will be found something

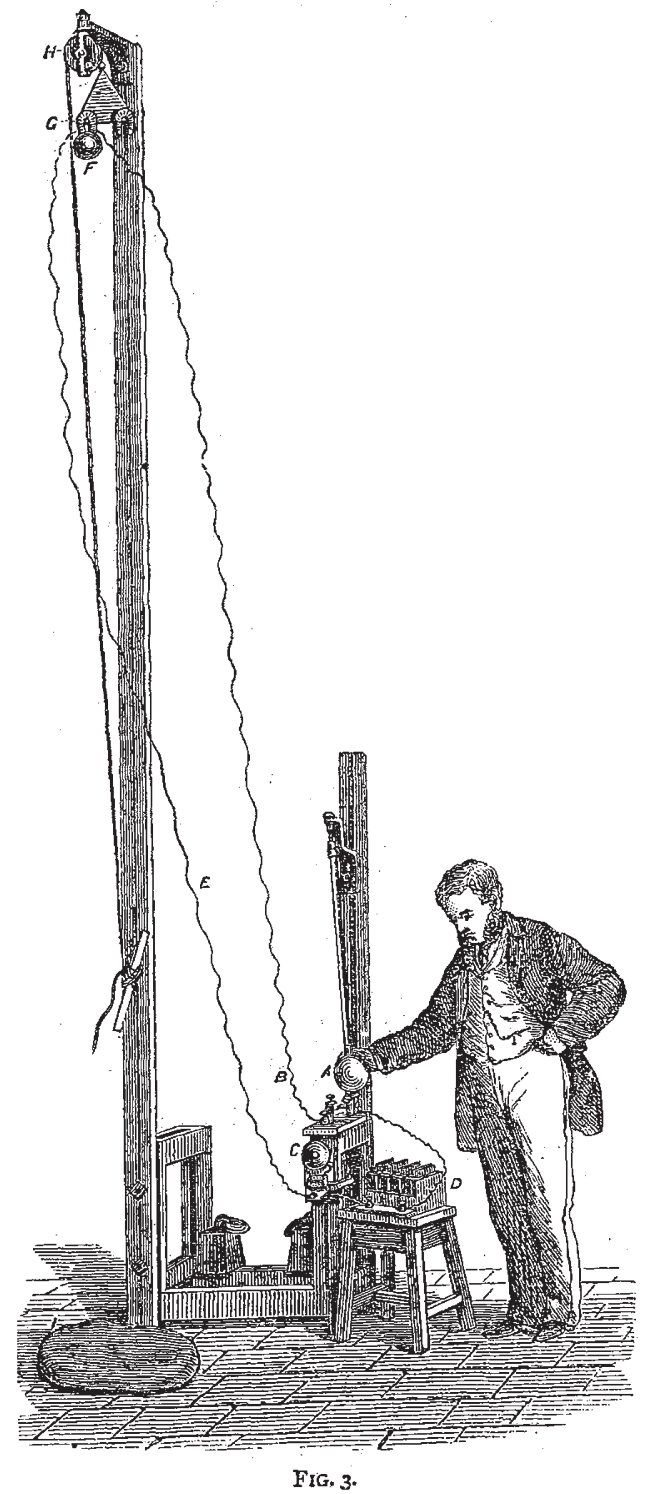

more. On the whole the work is one that will amply repay perusal, both by teacher and student, and is a most valuable supplement to works on the theory of mechanics. Nor must we take leave of the volume without adding that its general appearance-due to paper, printing, and illustrations - is truly beautiful, and, in fact, we cannot call to mind any English book of the same class which will bear comparison with it in these respects.

\section{J. F. TWISDEN}

ON THE BEST FORM OF COMPOUND PRISM FOR THE SPECTRUM MICROSCOPE

$\mathrm{N}$ studying the spectra of coloured solutions and solid 1 substances by means of the spectrum microscope, it is most important to employ prisms having a suitable amount of dispersion. It would be a very great mistake to suppose that the result is better with a very wide dis. persion. This, of course, makes the spectrum larger, but very greatly impairs the definition of the absorption-bands. Everyone who has had experience with an ordinary microscope must be well aware that a particular magnifying power is best for each particular class of object or kind of structure, and that in some cases nearly all the important characters would be lost by employing too high a power; but at the same time too low a power would be equally disadvantageous in other respects. This analogy holds good in the case of the dispersion of prisms. The power ought to be regulated by the character of the $a b-$ sorption-bands. If they are dark, narrow, well-defined, and lie close together, as in the case of partially opaque crystalline blow-pipe beads of borax containing deposited crystals of oxide of lanthanum with oxide of didymium, a somewhat powerful dispersion is not only admissible, but quite necessary to separate some of the bands. If, however, they are broad and faint like those seen in the spectra of many of the colouring matters found in animals and plants, a powerful dispersion spreads them over such a wide space, and makes the shading off so gradual, that the eye can scarcely appreciate the extra amount of absorption; whereas, when a lower dispersive power is used, a well-marked absorption-band can easily be seen. This is more especially the case with impure mixtures. I have found that when it was requisite to examine a mixed, somewhat turbid, coloured solution to detect, if possible, the presence of some substance which, when alone, gave a spectrum with distinct absorption-bands, no trace could be recognised by means of a prism of high dispersive power; but it could be detected without any difficulty with a lower. In carrying on practical investigations it is far more important to be able to succeed in such a case than to exhibit on a large and more imposing scale the spectra of a few substances which give dark and welldefined bands. There can be no doubt that it is a great advantage to have a number of prisms of different dispersive power, so that in all cases the most suitable may be used; but at the same time some observers might not wish to have more than one, and thus it becomes important to decide what amount of dispersion is the best for the generality of abjects-is sufficiently great to divide narrow, closely-placed bands, and yet not so great as to prevent our seeing broad and fairter. No magnifying power whatever is applied to the spectrum itself in the instrument now under consideration.

As described in some of my former papers, ${ }^{*}$ the compound, direct-vision prisms first made for me by $\mathrm{Mr}$. Browning were composed of two rectangular prisms of not very dense flint glass, and three of crown glass, one being rectangular, and the others of an angle of about $75^{\circ}$. This combination gives a dispersive power, which shows faint bands very well; but is not enough to divide the narrow and close bands seen in the spectra of a few substances. Mr. Browning then made prisms of similar construction, only that very dense flint glass was employed; This combination gives about double the former dispersion, which divides narrow and close bands admirably, but sometimes shows broad and fainter bands so very badly that they can scarcely be recognised. It thus appeared to me that, if only one compound prism be supplied with the instrument, the best dispersive power would be intermediate between these two extremes. At the same time much would depend on the particular purpose to which the instrument was applied, and also, to some extent, on the individual differences between different observers.

Mr. Browning has described the plan that he proposes for the measurement of the position of absorptionbands by means of a bright line, seen by reflection from the surface of the prism, moved backwards and forwards

* Popular Science Reviezv, vol. v., x866, pp. 66-77; Brit. As. Report, I 865 (pt. 2), p. II.

t Monthly Microscopical Fournal, vol, iii. p. 68. 